The committee sent four common organisms to 154 laboratories. From almost a half at least one of the sensitivities reported was wrong. Two-thirds of the errors were due to three well-known difficulties: the notorious problems of sulphonamide-sensitivity tests; failure to recognize the difference in the appearance of the zones produced by penicillin-sensitive and penicillinase-producing staphylococci; and difficulties with proteus strains. All the errors with penicillin were false reports of the sensitivity of Staphylococcus aureus; all the errors with sulphonamide were false reports of the resistance of Escherichia coli; and almost all the errors with ampicillin were false reports of the resistance of Proteus mirabilis. The committee analyses a number of factors contributing to these errors, but does not comment on the relative sizes of the laboratories taking part. Laboratories handling small numbers of specimens tend to be at a disadvantage in having fewer built-in controls, less experience, and often more dependence on commercial reagents, which, with small demands, have more time to deteriorate.

In its recommendations the committee reinforces a number of points which have been made elsewhere. Firstly, it suggests that known sensitive and known resistant organisms should regularly be included in tests. Secondly, it discourages multiple tests that are unrelated to the likely sensitivities of the organism (particularly where this includes potentially toxic antibiotics, such as chloramphenicol). And thirdly, it supports the use of sensitivity tests on the plate used for primary isolation of the organism. This last practice, once railed against and now properly coming into favour again, saves time, labour, and materials. It sometimes also requires a little extra skill in reading - and in the committee's survey one-quarter of the errors were made not in technique but in interpretation.

There is no doubt that delays are common in reporting sensitivity tests and that considerable waste and confusion result from testing commensals and contaminants. There is equally no doubt that delay is less serious if the result, when it arrives, is wrong. The only result the clinician needs quickly is the right one.

\section{Subacute Sclerosing Panencephalitis}

Mental retardation, epilepsy, and various disorders of movement are common enough in children. They are usually static or tend towards improvement. But in the rare cases of progressive cerebral degeneration of childhood the doctor becomes aware after the passage of some time of definite progression in one or other of these symptoms. The exact classification of these uniformly fatal conditions is largely a matter for the neuropathologist.

One rare disorder, intermediate in its clinical characteristics between the progressive cerebral degenerations and the acute

\footnotetext{
1 Metz, H., Gregoriou, M., and Sandifer, P., Arch. Dis. Childh., 1964, 39, 554

Pampiglione, G., ibid., 1964, 39, 558.

Dawson, J. R., Arch. Neurol. Psychiat., 1934, 31, 685.

Bogaert, L. van, f. Neurol. Neurosurg. Psychiat., 1945, 8, 101.
}

encephalitides, can often be diagnosed at sight. ${ }^{1}$ A child, usually of school age, makes, with rhythmic regularity, a stereotyped involuntary movement of distinctive type. A limb is abruptly raised. For a few seconds it remains frozen in elevation. Then slowly the movement melts away. Some eight seconds later the sequence is repeated. The diagnosis of subacute sclerosing panencephalitis can now be made. Electroencephalographic tracings show recurrent highamplitude slow-wave complexes imposed on an otherwise featureless record. By use of polymyography (with surface electrodes $)^{2}$ the E.E.G. abnormality may be shown closely to follow, precede, or coincide with the muscle action potentials, suggesting that both are driven by some third rhythmic change. The abnormal movements are not always so obvious, and in sleep they are modified or even absent, though the rhythmic E.E.G. abnormality persists. In the early case regularity may be less exact and the movements less frequent. Episodic staggering or clumsiness of hand movements may be all that is seen. The motor phenomena may be purely inhibitory (" antispasms"). Short-lived akinetic episodes may lead to the balance of a limb being suddenly and briefly impaired.

Other abnormalities become apparent during the evolution of the disease. Progressive intellectual deterioration may be followed by focal paralyses, and finally universal spastic paresis supervenes, while the disorders of movement may die away. The outcome is fatal for all but a few patients, in whom the disease becomes arrested at an advanced stage of disability.

The cerebrospinal fluid may contain a slightly raised amount of total protein, which contains an abnormally high level of gamma-globulin despite the lack of a corresponding level in the serum. There is a "paretic" type of colloidal gold curve. The negative Wassermann reaction goes against the diagnosis of neurosyphilis, but similar changes may occur in multiple sclerosis.

At necropsy the typical though not invariable finding is of acidophil hyaline nuclear inclusions in neurones and neuroglia. A variable amount of demyelination may also be noted. The changes are maximal in the posterior parts of the cerebral hemispheres, the cerebellar cortex, the medial thalamus, and certain brain-stem nuclei, notably in the olivo-pontinecerebellar system.

The condition was first described in its rapidly evolving form by J. R. Dawson, ${ }^{3}$ and then as more gradually progressive by $\mathrm{L}$. van Bogaert. ${ }^{4}$ The term subacute sclerosing panencephalitis is now accepted as covering a number of eponymous variants, which together constitute a distinctive entity. Its cause is unknown. The presence of inclusion bodies suggests virus invasion, but culture and serological testing are persistently negative. As in so many other conditions, the possibility of an autoimmune reaction has been raised.

Sir Hector MacLennan has been re-elected President of the Royal College of Obstetricians and Gynaecologists. 